\title{
Detection of very small neutrino masses in double-beta decay using laser tagging
}

\author{
M. Danilov, ${ }^{\mathrm{d}}$ R. DeVoe, ${ }^{\mathrm{c}}$ A. Dolgolenko, ${ }^{\mathrm{d}}$ G. Giannini, ${ }^{\mathrm{i}}$ \\ G. Gratta, ${ }^{\text {h P. Picchi, }}{ }^{\text {e }}$ A. Piepke, ${ }^{\text {a }}$ F. Pietropaolo, ${ }^{\mathrm{g}}$ P. Vogel,,${ }^{\mathrm{b}}$ \\ J-L. Vuilleumier, ${ }^{\mathrm{f}}$ Y-F. Wang, ${ }^{\mathrm{h}}$ O. Zeldovich ${ }^{\mathrm{d}}$ \\ a Physics Department, University of Alabama, Tuscaloosa AL, USA \\ ${ }^{\mathrm{b}}$ Physics Department, Caltech, Pasadena CA, USA \\ ${ }^{\mathrm{c}}$ Almaden Research Center, IBM, San Jose CA, USA \\ d ITEP, Moscow, Russia \\ e INFN, Laboratori Nazionali di Frascati, Italy \\ Istituto di Cosmogeofisica del CNR, Torino, Italy \\ Dipartimento di Fisica, Università di Torino, Italy \\ ${ }^{\mathrm{f}}$ Institut de Physique, Universite de Neuchatel, Switzerland \\ ${ }^{\circ}$ INFN, Sezione di Padova, via Marzolo 8, Padova, Italy \\ ${ }^{\mathrm{h}}$ Physics Department, Stanford University, Stanford CA, USA \\ i Dipartimento di Fisica, Università di Trieste, Italy
}

\begin{abstract}
We describe an approach to the study of neutrino masses that combines quantum optics techniques with radiation detectors to obtain unprecedented sensitivity. With it the search for Majorana neutrino masses down to $\sim 10 \mathrm{meV}$ will become accessible. The experimental technique uses the possibility of individually detecting $\mathrm{Ba}^{+}$-ions in the final state of ${ }^{136} \mathrm{Xe}$ double-beta decay via resonant excitation with a set of lasers aimed at a specific location in a large Time Projection Chamber. The specificity of the atomic levels provides tagging and, together with more traditional event recognition parameters, greatly suppresses radioactive backgrounds.
\end{abstract}

Key words: Double beta decay, Neutrino mass, TPC, optical tagging, ion trap 


\section{Introduction}

Recent results from a number of independent experiments [1] can be interpreted as due to finite neutrino masses and, in particular, high statistics measurements of atmospheric neutrinos by the Super-Kamiokande[2] experiment are regarded by most as firm evidence that neutrinos have non-zero masses.

While these measurements, based on oscillations, have set the stage for a systematic study of the intrinsic neutrino properties, only upper-limits exist on the absolute magnitude of neutrino masses. Indeed, theoretical models span a large range of scenarios, from the degenerate case where mass differences among flavors are small with respect to the absolute mass scale [3], to the hierarchical, where mass differences are of the same order as the mass themselves. However, while the neutrino mass scale is unknown, the present data on oscillations lead rather naturally to masses in the range $0.01<m_{\nu}<1 \mathrm{eV}$, as shown recently, e.g. in [4].

It is unlikely that direct neutrino mass measurements, most notably with tritium [5], will be able to reach sensitivities substantially below $1 \mathrm{eV}$ in the near future. In contrast, we will show that a large double- $\beta$ decay experiment using isotopically enriched ${ }^{136} \mathrm{Xe}$ can reach a sensitivity corresponding to neutrino masses as low as $\sim 0.01 \mathrm{eV}$. A xenon detector offers the unique possibility of identifying the final state, thus providing an essentially background-free measurement of unprecedented sensitivity for the neutrino mass.

It is well known that neutrinoless double beta decay, $0 \nu \beta \beta$, can proceed only if neutrinos are massive Majorana particles. If the $0 \nu \beta \beta$ occurs, the effective Majorana neutrino mass $\left\langle m_{\nu}\right\rangle$ is related to the half-life $T_{1 / 2}^{0 \nu \beta \beta}$ as:

$$
\left\langle m_{\nu}\right\rangle^{2}=\left(T_{1 / 2}^{0 \nu \beta \beta} G^{0 \nu \beta \beta}\left(E_{0}, Z\right)\left|M_{G T}^{0 \nu \beta \beta}-\frac{g_{V}^{2}}{g_{A}^{2}} M_{F}^{0 \nu \beta \beta}\right|^{2}\right)^{-1}
$$

where $G^{0 \nu \beta \beta}\left(E_{0}, Z\right)$ is a known phase space factor depending on the end-point energy $E_{0}$ and the nuclear charge $Z$, and $M_{G T}^{0 \nu \beta \beta}$ and $M_{F}^{0 \nu \beta \beta}$ are the Gamow-Teller and Fermi nuclear matrix elements for the process. Here we have defined $\left\langle m_{\nu}\right\rangle=\sum_{i} m_{i} U_{e i}^{2}$ with $U$ being the mixing matrix in the lepton sector, and $m_{i}$ the masses of the individual Majorana neutrinos. Hence, although difficulties in the nuclear models used to calculate the matrix elements give some uncertainty on the value of $\left\langle m_{\nu}\right\rangle$ (see, e.g. [6]), $0 \nu \beta \beta$ decay is sensitive to the masses of all neutrino flavors, provided that the mixing angles are non-negligible. 


\section{Backgrounds and Experimental Limitations}

At present the best sensitivities to $0 \nu \beta \beta$ decay have been reached with ${ }^{76} \mathrm{Ge}$ diode ionization counters with an exposure of $24.16 \mathrm{~kg}$ yr [7] and with a ${ }^{136} \mathrm{Xe}$ time projection chamber (TPC) with an exposure of $4.87 \mathrm{~kg}$ yr [8]. The measured half-life limits of $5.7 \times 10^{25}$ yrs for germanium and $4.4 \times 10^{23}$ yrs for xenon can be interpreted as neutrino mass limits of, respectively, $\left\langle m_{\nu}\right\rangle<0.2(0.6) \mathrm{eV}$ and $\left\langle m_{\nu}\right\rangle<2.2(5.2) \mathrm{eV}$ using the Quasi-Particle Random Phase Approximation (QRPA) [9] (Shell Model (NSM) [10]) for the nuclear matrix element calculation.

The germanium detector rejects background on the basis of its excellent energy resolution and of pulse-shape discrimination [7] and has recently reported a specific background as low as $0.3 \mathrm{~kg}^{-1} \mathrm{yr}^{-1} \mathrm{FWHM}^{-1}$. The relatively inferior energy resolution in TPCs have been complemented by superior tracking capabilities that allow the xenon experiment to partially reconstruct the two-electron topology of $\beta \beta$-decay, obtaining a specific background of $2.5 \mathrm{~kg}^{-1} \mathrm{yr}^{-1} \mathrm{FWHM}^{-1}$ [8]. None of these backgrounds is sufficient for a decisive experiment in the interesting region discussed above. For that, it is essential to find a reliable way to drastically increase the size of the detectors while, simultaneously, reducing the backgrounds. This dual requirement stems from the fact that in a background-free experiment the neutrino mass sensitivity scales as $\left\langle m_{\nu}\right\rangle \propto 1 / \sqrt{T_{1 / 2}^{0 \nu \beta \beta}} \propto 1 / \sqrt{N t}$, where $t$ is the measurement time and $N$ the number of nuclei under study. In the opposite extreme, when the background scales with $\mathrm{Nt}$, the neutrino mass sensitivity would scale only as $\left\langle m_{\nu}\right\rangle \propto 1 /(N t)^{1 / 4}$. Obviously, the backgrounds observed in all current experiments will become the true limiting factor of any kind of future large experiment, hampering the full utilization of very large masses of $\beta \beta$ emitters. Unlike other isotopes, however, ${ }^{136} \mathrm{Xe}$ allows for direct tagging of the Ba-ion final state using optical spectroscopy, as pointed-out for the first time in [11]. While this technique cannot discriminate between $0 \nu \beta \beta$ and $2 \nu \beta \beta$-decays, this second process is not the dominant background at the sensitivities sought here and can be eliminated by kinematical reconstruction in a xenon TPC. Moreover, xenon is one of the easiest isotopes to enrich, and like argon can be used as active medium in ionization chambers. Hence, an experiment with xenon can use an entirely new variable in order to drastically reduce the backgrounds and explore the range of interesting neutrino masses.

\section{Barium detection in xenon}

The $\beta \beta$-decay of ${ }^{136} \mathrm{Xe}$ produces a $\mathrm{Ba}^{++}$ion in the final state that can be readily neutralized to $\mathrm{Ba}^{+}$by an appropriate secondary gas in the TPC. The barium ion can 
then be individually detected through its laser-induced fluorescence. Single $\mathrm{Ba}^{+}$ions were first observed in 1978 [12] using a radio frequency quadrupole trap and laser cooling.

The level structure of the alkali-like $\mathrm{Ba}^{+}$ion is shown in Figure 1. Due to the strong

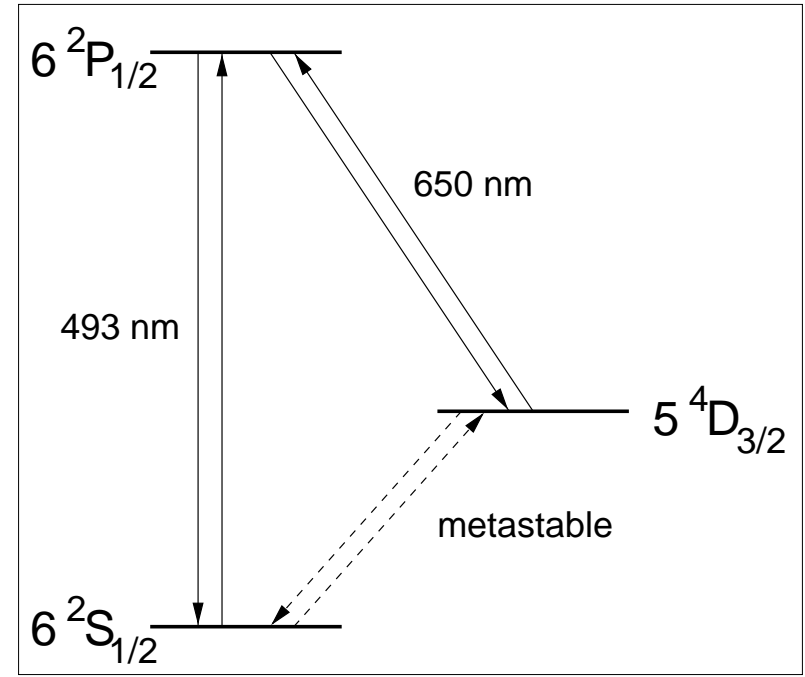

Fig. 1. Atomic level scheme for $\mathrm{Ba}^{+}$ions.

$493 \mathrm{~nm}$ allowed transition ground-state ions can be optically excited to the $6^{2} \mathrm{P}_{1 / 2}$ state from where they have substantial branching ratio $(30 \%)$ to decay into the metastable $5^{4} \mathrm{D}_{3 / 2}$ state. Specific $\mathrm{Ba}^{+}$detection is then achieved by exciting the system back into the $6^{2} \mathrm{P}_{1 / 2}$ state with $650 \mathrm{~nm}$ radiation and observing the blue photon from the decay to the ground state ( $70 \%$ branching ratio). This transition has a spontaneous lifetime of $8 \mathrm{~ns}$ and when saturated will radiate $6 \times 10^{7}$ photons $/ \mathrm{s}$. A pair of lasers tuned onto the appropriate frequencies and simultaneously steered to the place where the $\beta \beta$-decay candidate event is found can provide a very selective validation, effectively providing a new independent constraint to be used in $\beta \beta$-decay background subtraction. The light from the $\mathrm{P}$ to $\mathrm{S}$ transition conveniently lays in the region of maximum quantum efficiency of bialkali photocathodes so that an array of conventional large-area photomultipliers can be used for the detection. The very large saturation rate makes the experiment possible even with modest photocathode angular coverage. While it is possible in principle to steer the lasers anywhere inside the TPC, the very large volume and the need for light baffling may favor a scheme in which the $\mathrm{Ba}^{+}$drift in the large electric field is used to bring the ion in specific laser detection regions.

The primary difference between the single atom work done previously and this experiment is that here the $\mathrm{Ba}^{+}$ion is not in vacuum but rather in a buffer gas $(\mathrm{Xe})$ at a pressure of several atmospheres. The high pressure xenon has two effects: first, it effectively traps the barium since diffusion in the dense gas is sufficient to confine the atoms for long enough time to obtain a signal; second, it pressure broadens the 
optical transitions increasing the laser power required. We calculate that at $5 \mathrm{~atm}$ the barium ion will diffuse only $\simeq 0.7 \mathrm{~mm}$ in $1 \mathrm{~s}$, and during this time it can be cycled over $10^{7}$ times through the three-level scheme of Figure 1, emitting more than $10^{7} 493 \mathrm{~nm}$ photons. The ion drift in the electric field of the TPC can be accurately measured and corrected for in the process of steering the lasers. For the moment we notice that drift velocities of $\mathrm{Tl}^{+}$in Xe have been measured [13] and are low enough to make the correction possible. We also find from preliminary calculations that at the same pressure the broadened line-width is $\sim 20 \mathrm{GHz}, 1000$ times greater than the natural line-width. This results in a saturation intensity of $\sim 5 \mathrm{~W} / \mathrm{cm}^{2} . \mathrm{Ba}^{+}$ lifetime, together with pressure broadening of the lines involved, and the lifetime of the $5^{4} \mathrm{D}_{3 / 2}$ state, will have to be measured in xenon before the detection system can be fully optimized. However, measurements on the corresponding neutral Ba states at $0.5 \mathrm{~atm}$ in He and Ar [14] show that operations at 5 to $10 \mathrm{~atm}$ should be possible.

\section{A large xenon gas TPC with barium tagging}

Among the different gas ionization detectors known, a TPC is the ideal detector for $\beta \beta$-decay as it has no wires or other materials in the detection volume hence reducing the background internal to the detector and simplifying the laser scanning. The requirements of size, efficiency for contained events, energy, and spatial resolution can be achieved with a large $\left(40 \mathrm{~m}^{3}\right)$ gas-phase TPC with xenon. TPCs of similar size at atmospheric pressure are successfully in operation $[15,16]$, in one case with some $10 \mathrm{~kg}$ of ${ }^{136} \mathrm{Xe}$ [17]. A 5 to $10 \mathrm{~atm}, 40 \mathrm{~m}^{3}$ chamber would contain 1 to 2 tons of ${ }^{136} \mathrm{Xe}$ and is within the reach of current state-of-the-art technology.

The ultimate sensitivity of the technique is probably limited by the practical availability of ${ }^{136} \mathrm{Xe}$ that represents $8.9 \%$ of natural xenon and has to be extracted by isotopic separation. While the isotopic separation is indeed one of the main challenges of the experiment, in the case of ${ }^{136} \mathrm{Xe}$ this operation is simplified by the fact that xenon is a gas at standard conditions, and essentially any known separation technology can be used. Relatively large amounts of ${ }^{136}$ Xe have been obtained in the past by ultracentrifugation [18] with purities sufficient for the experiment described [19]. ${ }^{136} \mathrm{Xe}$ handling is simplified by its inert character that allows for the extraction of cosmogenically produced elements with distillation and chemical filtering that can be carried-on in the underground laboratory prior (and during) the operation of the experiment. An experiment with up to 10 tons of ${ }^{136} \mathrm{Xe}$ is consistent with the availability of xenon on the world market [20] and can be setup by assembling a few TPC modules inside a single pressure vessel or, possibly, by increasing the pressure and/or volume of a single chamber. 
The use of liquid-xenon (LXe) in a TPC would result in a very compact detector with considerable advantages. However the range of $1.2 \mathrm{MeV}$ electrons from the $0 \nu \beta \beta$ decay in LXe is only of $2.4 \mathrm{~mm}$ so that, given any conceivable spatial resolution, the topological information, essential for background rejection, would be lost. In contrast, at room temperature 5 atm of xenon gas correspond to a density of $30 \mathrm{~g} / \mathrm{l}$ and an electron range of $21.6 \mathrm{~cm}$ at $1.2 \mathrm{MeV}$. In Figure 2 we show a possible scheme for the $\beta \beta$-decay detector.

It is known [21] that it is quite difficult to obtain electron multiplication and stable operation in xenon chambers. This is due to the far UV scintillation light $\left(\lambda_{\text {scint }} \simeq 178 \mathrm{~nm}\right.$ corresponding to $6.93 \mathrm{eV}$ ), copiously produced with the multiplication process, that in turn ionizes the gas and extracts electrons from the metallic surfaces. In our case this particular requirement is greatly relaxed by the use of gas micropattern detector [22] planes instead of a more conventional anode wire array for the chamber readout, drastically limiting the solid angle available for the UV radiation [23]. In addition, the chamber will also use a quencher gas, able to absorb the xenon scintillation UV and re-emit light in the blue or green so that timing information can be recovered using the photomultipliers and the event can be localized also in the third (time) coordinate. A field of $\sim 1 \mathrm{kV} / \mathrm{cm}$ will be needed in order to achieve high drift velocity for electrons in xenon. While this field strength is not sufficient to neutralize $\mathrm{Ba}^{++}$ to $\mathrm{Ba}^{+}$, an appropriate choice of ionization potential (IP) for the quencher gas can achieve this purpose. IPs between $5.2 \mathrm{eV}(\mathrm{Ba})$ and $10.001 \mathrm{eV}\left(\mathrm{Ba}^{+}\right)$are found in several organic molecules that would provide a stable environment for $\mathrm{Ba}^{+}$. Molecules like TMA $\left(\left(\mathrm{CH}_{3}\right)_{3} \mathrm{~N}\right)$, TEA $\left(\left(\mathrm{C}_{2} \mathrm{H}_{5}\right)_{3} \mathrm{~N}\right)$, TMG $\left(\left(\mathrm{CH}_{3}\right)_{4} \mathrm{Ge}\right)$ and TMS $\left(\left(\mathrm{CH}_{3}\right)_{4} \mathrm{Si}\right)$ are candidates fulfilling the above requirements.

An underground site with an overburden of $\simeq 2000$ mwe (meter water equivalent) will reduce the cosmic ray muon flux to $<10^{-2} \mathrm{~m}^{-2} \mathrm{~s}^{-1}$, corresponding to less than $0.1 \mathrm{~Hz}$ through the detector. Muons recorded in the TPC have a distinctive signature and indeed were observed and rejected by the Gotthard group [8] based on track length, lack of scattering (high energy), and specific ionization. The last two features provide good discrimination even for tracks that clip a small corner of the chamber. The online trigger processor will be able to analyze and reject most muon tracks without activating the laser Ba-tagging system. Based on simple geometrical considerations we expect the laser system to be activated by muon tracks less than once per hour. Some background will be produced by neutrons produced by muon spallation in the structures (rock and other materials) outside the detector. These fast neutrons enter the detector with some efficiency and produce spallation reactions on the xenon, carbon and hydrogen nuclei contained in the TPC. While the original muon and the neutron trail go undetected, the spallation processes provide very distinct high ionization, short tracks and can be distinguished and rejected even at trigger level. Hence at the depth considered the detector does not need an active veto counter, as already found by the Gotthard group. 


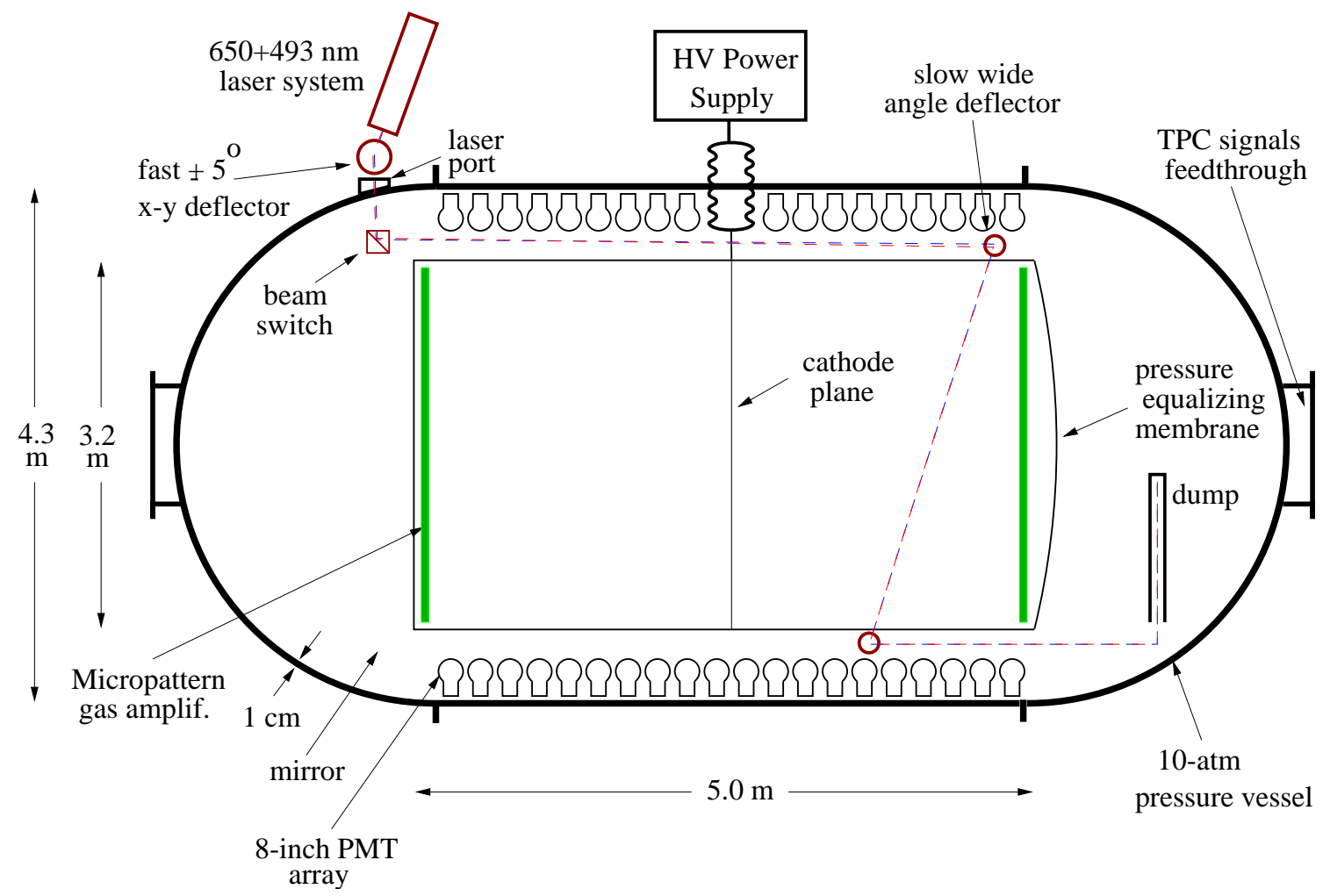

Fig. 2. Conceptual layout of the $\beta \beta$ detector. The active volume of ${ }^{136} \mathrm{Xe}$ is contained by a transparent acrylic cylinder. An inert buffer gas transfers the pressure to an outer steel vessel and insulates the high voltage sections of the TPC. The central cathode plane is held at a potential of $\approx 250 \mathrm{kV}$ while the electron multiplication and readout is achieved with micro-pattern gas amplifier [22] planes. An array of photomultiplier tubes detects the scintillation light from the xenon and the fluorescence from the Ba-ions. All the materials inside the pressure vessel are selected for low activity.

More serious are the $\gamma$-ray backgrounds from natural radioactivity, either produced outside the TPC (mainly by the rock or concrete), or inside (mainly ${ }^{222} \mathrm{Rn}$ and, possibly, ${ }^{85} \mathrm{Kr}$ and ${ }^{42} \mathrm{Ar}$ ). External $\gamma$-ray backgrounds from the rock and concrete can be attenuated by a $\sim 25 \mathrm{~cm}$ thick lead or steel enclosure and by the $1 \mathrm{~cm}$ thick pressure vessel that will be built out of low-activity steel. Additionally, cleaner shielding could be provided, if needed. In the following we conservatively assume that the TPC itself (without Ba-tagging system) will have the same specific rate of mis-identified background as the Gotthard experiment, and we rely on Ba-tagging for the final step of reduction. This hypothesis is conservative since the larger volume of our TPC provides better self shielding for the radiation produced externally. 


\section{Discussion}

We estimate the position and energy resolution of our chamber from an extrapolation using the Gotthard TPC. Assuming a drift velocity similar to the one in Gotthard and a drift distance of $250 \mathrm{~cm}$ (3.6 times larger than the Gotthard case) we obtain transverse and longitudinal position resolutions $(\sigma)$ of better than $5 \mathrm{~mm}$. This figure should give us roughly the same background suppression factor as obtained at Gotthard, since the electron range is $21 \mathrm{~cm}$. Furthermore, unlike in the case of the Gotthard experiment, the knowledge of the time of occurrence will provide longitudinal localization of each event, improving the understanding of external backgrounds and allowing for a longitudinal fiducial volume cut.

Energy resolution should be no worse than the $\sigma_{E} / E=2.5 \%$ (at $1.592 \mathrm{MeV}$ ) obtained at Gotthard, since in addition to the total charge, also the scintillation light will be collected [24]. The possibility of using the laser tagging system with a small spot-size in a "raster scan" mode around the event location, will localize the decay vertex with mm precision and allow a full kinematical fit including energy, angular correlation and range for each of the electrons. It is expected that substantial improvement in energy resolution and background rejection will be possible in this way.

While the quantitative advantage of each of the techniques discussed will be better understood after extensive laboratory tests and a full Monte Carlo simulation, here we simply assume that the above methods, together with the $\mathrm{Ba}^{+}$identification, will reduce the sum of all radioactivity backgrounds by at least three orders of magnitude with respect to the Gotthard case, making a 10-ton ${ }^{136}$ Xe experiment essentially background free. From the size and geometry of the chamber we obtain an efficiency for fully contained events of $70 \%$. In Table 1 we compare the projected sensitivity of this experiment with the present limits on $0 \nu \beta \beta$ decay.

It is interesting to note that for the very large isotopic masses contemplated here, the background on $0 \nu \beta \beta$ decay from the well known $2 \nu \beta \beta$ mode has to be carefully estimated. As already remarked this "background" is not directly suppressed by the laser tagging methods. However, the energy spectra of the electrons that represent the only distinctive feature of this background will be better measured thanks to the better knowledge of the event kinematics. In the following we will conservatively disregard this additional information and suppress the $2 \nu \beta \beta$ mode using total energy information, with the resolution mentioned above. In order to understand the role of the resolution we select events in the two intervals $I_{s y m}=\left[Q_{\beta \beta}-2 \sigma_{E}, Q_{\beta \beta}+2 \sigma_{E}\right]$ and $I_{+}=\left[Q_{\beta \beta}, Q_{\beta \beta}+2 \sigma_{E}\right]$. We then compute the number of $2 \nu \beta \beta$ decay events [6] left in each case. For $\sigma_{E} / E=2.8 \%$ we have 23 events $\mathrm{yr}^{-1}$ ton $^{-1}$ left in $I_{\text {sym }}$ and 0.36 events $\mathrm{yr}^{-1}$ ton $^{-1}$ left in $I_{+}$. A better resolution of $\sigma_{E} / E=2 \%$ is used in the 10 ton - 10 yr exposure in Table 1 . The loss in efficiency due to the asymmetric cut (and to the tails beyond $2 \sigma$ ) are taken into account in the table as appropriate. It is clear, however, that our estimate is quite conservative as the $2 \nu \beta \beta$ decay background 


\begin{tabular}{|l|c|c|c|c|c|c|c|c|}
\hline Isotope and & $\begin{array}{c}\text { Total } \\
\text { Reference }\end{array}$ & $\begin{array}{c}\text { Enrich. } \\
\text { gass } \\
(\mathrm{kg})\end{array}$ & $\begin{array}{c}\text { Det. } \\
(\%)\end{array}$ & $\begin{array}{c}\text { Meas. } \\
\text { eff. }\end{array}$ & $\begin{array}{c}\text { Bkgd. } \\
\text { time } \\
(\mathrm{yr})\end{array}$ & \multicolumn{2}{|c|}{$T_{1 / 2}^{0 \nu \beta \beta}$} & \multicolumn{2}{|c|}{$\left\langle m_{\nu}\right\rangle$} \\
QRPA & NSM \\
$(\mathrm{yr})$ & \multicolumn{2}{|c|}{$(\mathrm{eV})$} \\
\hline${ }^{76} \mathrm{Ge}[7]$ & 11 & 86 & 75 & 2.2 & 0.3 & $5.7 \times 10^{25}$ & 0.2 & 0.6 \\
${ }^{136} \mathrm{Xe}[8]$ & 3.3 & 63 & 22 & 1.47 & 2.5 & $4.4 \times 10^{23}$ & 2.2 & 5.2 \\
${ }^{136} \mathrm{Xe}$ projected & 1000 & 65 & 70 & 5 & $0^{*}+$ & $8.3 \times 10^{26}$ & 0.051 & 0.14 \\
$\left(\right.$ for $\left.\sigma_{E} / E=2.8 \%\right)$ & & & & & 1.8 events & & & \\
${ }^{136} \mathrm{Xe}$ projected & 10000 & 65 & 70 & 10 & $0^{*}+$ & $1.3 \times 10^{28}$ & 0.013 & 0.037 \\
$\left(\right.$ for $\left.\sigma_{E} / E=2.0 \%\right)$ & & & & & 5.5 events & & & \\
\hline
\end{tabular}

Table 1

Comparison of the best present double-beta decay experiments and the project described here. The technique of $\mathrm{Ba}^{+}$tagging will reduce the background, given in units of $\mathrm{kg}^{-1} \mathrm{yr}^{-1} \mathrm{FWHM}^{-1}$ if not noted otherwise, to the level necessary to fully utilize the large mass of isotopic species. All other experimental parameters are assumed to be the same as in the Gotthard experiment. We list here, together, the case of an initial detector with 1 ton of ${ }^{136} \mathrm{Xe}$ and the final results possible with 10 tons of ${ }^{136} \mathrm{Xe}$ and a very long (10 years) data-taking period. The quantities marked with * are radioactivity backgrounds that are assumed to be negligible as discussed in the text. In addition the background from misidentified $2 \nu \beta \beta$ decays is also shown in total events in each exposure using the asymmetric analysis interval described in the text.

will be further suppressed by the kinematic fitting.

The projected neutrino mass sensitivity of $10-50 \mathrm{meV}$ would make the discussed experiment competitive with other large scale double beta searches which have been proposed. NEMO3 is scheduled to start data taking in 2001 with initially $7 \mathrm{~kg}$ of ${ }^{100} \mathrm{Mo}$ and $1 \mathrm{~kg}$ of ${ }^{82} \mathrm{Se}$ in form of passive source foils in a gas tracking detector utilizing a magnetic field and a scintillator calorimeter [25]. A neutrino mass sensitivity of $0.1 \mathrm{eV}$ is expected after 5 years. The ultimate goal is to run with $20 \mathrm{~kg}$ of ${ }^{82} \mathrm{Se},{ }^{100} \mathrm{Mo}$ or ${ }^{150} \mathrm{Nd}$ in order to measure an effective neutrino mass below $0.1 \mathrm{eV}$. A large cryogenic detector (CUORE), able to operate $600 \mathrm{~kg}$ of $\mathrm{TeO}_{2}$ crystals, could be used to search for the $0 \nu \beta \beta$ decay of ${ }^{130} \mathrm{Te}$. A neutrino mass sensitivity of around $0.1 \mathrm{eV}$ has been quoted for this device [26]. The proponents of the GENIUS project propose to use up to one ton of isotopically enriched ${ }^{76} \mathrm{Ge}$ suspended in a large tank of liquid nitrogen [27]. The goal is to reach a mass sensitivity of around $0.01 \mathrm{eV}$. The latter two are calorimetric approaches. However, it has to be pointed out that the experiment discussed in this paper is the only one among those third generation projects which plans to utilize a novel technique for background suppression in the form of Ba tagging.

In summary, we have described an advanced $\beta \beta$ detector system that uses a hybrid 
of atomic and particle physics techniques to qualitatively improve background suppression. Such detector opens new possibilities in using massive amounts of ${ }^{136} \mathrm{Xe}$ for an advanced $\beta \beta$-decay experiment that would explore neutrino masses in the range 10 - $50 \mathrm{meV}$, providing a unique opportunity for discoveries in particle physics and cosmology.

\section{Acknowledgments}

We would like to specially thank F. Boehm for the help and guidance received in formulating much of this paper. We also owe gratitude to U. Becker, S. Chu, H. Henrikson, L. Ropelewski, T. Thurston and R. Zare for many useful discussions. Finally

we would like to thank R.G.H. Robertson for pointing out an inconsistency in the original version of Table 1.

\section{References}

[1] See e.g. Neutrino section in C. Caso et al., Eur. Phys. J. C 3 (1998) 1.

[2] Y. Fukuda et al., Phys. Rev. Lett. 81 (1998) 1562.

[3] D.O. Caldwell and R.N. Mohapatra, Phys. Rev. D 48 (1993) 3259;

J. Ellis and S. Lola, Phys. Lett. B 458 (1999) 310;

V. Barger and K. Whisnat, Phys. Lett. B 456 (1999) 194.

[4] S.M. Bilenkii et al., Phys. Lett. B 465 (1999) 193.

[5] P. Fisher, B. Kayser and K. McFarland, Ann. Rev. Nucl. Part. Sci. 49 (1999) 481.

[6] P. Vogel to appear in "Current aspects of Neutrino Physics" D.O. Caldwell ed., Springer, to appear in 2000.

[7] L. Baudis et al., Phys. Rev. Lett. 83 (1999) 41.

[8] R. Luescher et al., Phys. Lett. B 434 (1998) 407.

[9] A. Staudt et al., Europhys. Lett. 13 (1990) 31.

[10] E. Caurier et al., Phys. Rev. Lett. 77 (1996) 1954.

[11] M.K. Moe, Phys. Rev. C 44 (1991) R931.

[12] W. Neuhauser, M. Hohenstatt, P. Toschek, and H. Dehmelt, Phys. Rev. Lett. 41 (1978) 233. 
[13] T.M. Maddern and L.W. Mitchell, Nucl. Inst. and Meth. A 359 (1995) 506.

[14] E. Erlacher and J. Hunnekens, Phys. Rev. A 46 (1992) 2642 (1992).

[15] C. Brand et al., Nucl. Inst. and Meth. A 283 (1989) 567.

[16] W.B. Atwood et al., Nucl. Inst. Meth. A 306 (1991) 446.

[17] V.A. Artemiev et al., Nucl. Inst. and Meth. A303 (1991) 309;

V. Artemiev et al., Phys. Lett. B 345 (1995) 564.

[18] W.L. Roberts Nucl. Inst. and Meth. A 282 (1989) 271.

[19] E. Bellotti et al. Nucl. Inst. and Meth. B 62 (1992) 529.

[20] P.K. Lebedev and V.I. Pryanichnikov, Nucl. Inst. and Meth. A 327 (1993) 222.

[21] F. Sauli, CERN Yellow Report 77-09, Geneva 3 May 1977.

[22] For a general review on gas micro-pattern detectors see: F. Sauli and A. Sharma, Ann. Rev. Nucl. Part. Sci. 49 (1999) 341.

[23] A. Bressani et al., Budker Institute Preprint INP-98-59, Novosibirsk 1998.

[24] J. Séguinot et al., Nucl. Inst. and Meth. A 354 (1995) 280.

[25] NEMO3 proposal, LAL preprint 94-29 (1994);

C. Marquet for the NEMO coll., to appear in Nucl. Phys. B (Proc.Suppl) 87 (2000).

[26] E. Fiorini, Phys. Rep. 307 (1998) 309.

[27] L. Baudis et al., Phys. Rep. 307 (1998) 301. 